\title{
A X-ray Excitable Vibrational AIE System Based on Platinum
}

\section{(II) Salts}

Zheng Zhao ${ }^{1,2 \#}$, Zaiyu Wang ${ }^{1, \#}$, Javad Tavakoli ${ }^{3}$, , Chen Peng ${ }^{1}$, Yu Xiong ${ }^{2}$, Youhong Tang $^{3^{*}}$, Jacky. W. Y. Lam ${ }^{1}$, Ben Zhong Tang ${ }^{1,2,5^{*}}$

${ }^{1}$ Department of Chemistry, The Hong Kong University of Science and Technology, Clear Water Bay, Kowloon, Hong Kong, China. ${ }^{2}$ HKUST Shenzhen Research Institute, No. 9 Yuexing 1st RD, South Area Hi-tech Park, Nanshan, Shenzhen 518057, China. ${ }^{3}$ Medical Device Research Institute, College of Science and Engineering, Flinders University, Adelaide, South Australia 5042, Australia. ${ }^{4}$ State Key Laboratory of Supramolecular Structure and Materials College of Chemistry Jilin University. ${ }^{5}$ NSFC Center for luminescence from Molecular Aggregates, SCUT-HKUST Joint Research Institute, State Key Laboratory of Luminescent Materials and Devices, South China University of Technology, Guangzhou 510640, China.

Abstract: Aggregation-induced emission luminogen excited by X-ray is highly advantageous for imaging and diagnosis but has not been achieved. Herein, we demonstrated $\mathrm{BaPt}(\mathrm{CN})_{4}$ as the first inorganic salt based AIE system which can be excited by X-ray. The AIE mechanism of $\mathrm{BaPt}(\mathrm{CN})_{4}$ is studied and the X-ray luminescence of $\mathrm{BaPt}(\mathrm{CN})_{4}$ is tested.

\section{Introduction}

Molecular motion responsive luminescent materials have attracted great attention due to their significant roles in affording multifunctional materials and broadening the fundamental understanding of dynamic molecular systems. ${ }^{1-5}$ In this regard, one of the most fascinating concepts is aggregation-induced emission (AIE) proposed by Tang and co-workers. ${ }^{6,7}$ Typical AIE luminogens (AIEgens) show strong emission in aggregated/solid state but are non-emissive in their monodispersed state. Further underlying mechanism study unveiled that luminescence of AIE system basically depends on the intramolecular motion extent that influenced by the surrounding environment of the molecules. ${ }^{8}$ In monodispersed state, the active intramolecular motion including rotation and vibration accelerates the non-radiative decay process of the excitons and quenches the emission, while in the solid state or other media that can restriction the intramolecular motion, the non-radiative decay will be suppressed and consequently enhance the emission. The solid-state emission and environmental sensitivity of AIEgens enable them highly advantageous as nano imaging agents for long-term tracing and as sensors or imaging probes for biological species detection. ${ }^{8-12}$

So far, large amounts of AIEgens with different colors have been developed and successfully utilized for cell and microbial imaging, biomarker detection, physiological process monitoring and imaging guided surgery etc. ${ }^{5,6,9}$ However, an unignored limitation of these AIEgens is that their excitation wavelengths are mostly located in the UV/vis range, which has the disadvantages of significant auto-fluorescence resulting in poor signal-to-noise ratio, and poor tissues penetration depth. Although some near-infrared absorption and two-photo excitable AIEgens have been developed 
to improve this problem, they may suffer quite low luminescence quantum yield and still have limited penetrative power in living tissues. ${ }^{13-16}$

On the other hand, Luminogens excited by X-ray are attracting more and more attention recently since X-ray can easily penetrate biological tissues and thus improve both limitations of auto-fluorescence and poor penetration depth of common luminogens. ${ }^{17-24}$ For instance, the luminescence signal of some inorganic system can be detected even in $5 \mathrm{~cm}$ thick porcine tissues under X-ray irradiation. ${ }^{25}$ Furthermore, since X-ray computed tomography had been widely used in clinic for imaging the anatomy, luminogens with X-ray luminescence thus are advantageous for dual-modal $\mathrm{X}$-ray/X-ray excited fluorescence imaging wherein optical signal and anatomical information was simultaneously obtained. ${ }^{17}$ Therefore, it is highly advantageous to develop X-ray excitable AIEgens, which potentially integrated both the advantages of AIEgens and X-ray excitation and possibly open a new door for fluorescence imaging in bio-applications.

Platium (II) salts have been widely investigated as luminescent materials for optoelectronics and bio-imaging applications. ${ }^{26-30}$ While $\mathrm{Pt}(\mathrm{CN}) 4^{2-}$ complexes possibly are among the earliest luminescent Platium (II) salts, since their luminescence has been observed by Sir Stokes as early as in 1853, when the term "fluorescence" was coined. ${ }^{31}$ However, the luminescence phenomenon of these $\mathrm{Pt}(\mathrm{CN})_{4}{ }^{2-}$ complexes were not deeply investigated at that time possibly due to the limitation people's knowledge to luminescence. Until 1970s, scientists began to systematically study the electronic structure and spectroscopy properties of these $\mathrm{Pt}(\mathrm{CN}) 4^{2-}$ complexes and their derivatives. ${ }^{32-36}$ Currently, research interest in these inorganic complexes is continuing with new understanding and applications are continuously developed, a recent study just explored the application of $\mathrm{ZnPt}(\mathrm{CN})_{4}$ in ammonia detection. ${ }^{36,37}$ Since the research on luminescent materials and photophysical mechanism have made great achievements in the past four decades, re-investigation these old compounds based on current understanding on luminescence is interesting and of big scientific value, which possibly results in some new discovery or new understanding on these old luminescent materials. In this work, we found the inorganic salts, $\mathrm{BaPt}(\mathrm{CN})_{4}$ showed typical AIE characteristic, which showed no emission in water solution but bright luminescence in aggregates and solid state. Spectroscopy study indicates that the intramolecular vibration should be responsible for the non-luminescent characteristic of $\mathrm{BaPt}(\mathrm{CN})_{4}$ in solution state, while crystal structure analysis suggests multiple intermolecular hydrogen bonding and electrostatic interactions as well as Pt...Pt interactions contribute greatly to the solid state emission efficiency and emission wavelength. The influence of intermolecular Pt...Pt interactions on emission wavelength was further confirmed by hydrostatic pressure depended fluorescence experiment. More importantly, we found $\mathrm{BaPt}(\mathrm{CN})_{4}$ could be excited by X-ray and emit bright green luminescence. To the best of our knowledge, this is the first AIE system based on inorganic salts and also the first X-ray excitable AIEgen, which opens a new door for the AIE research. 

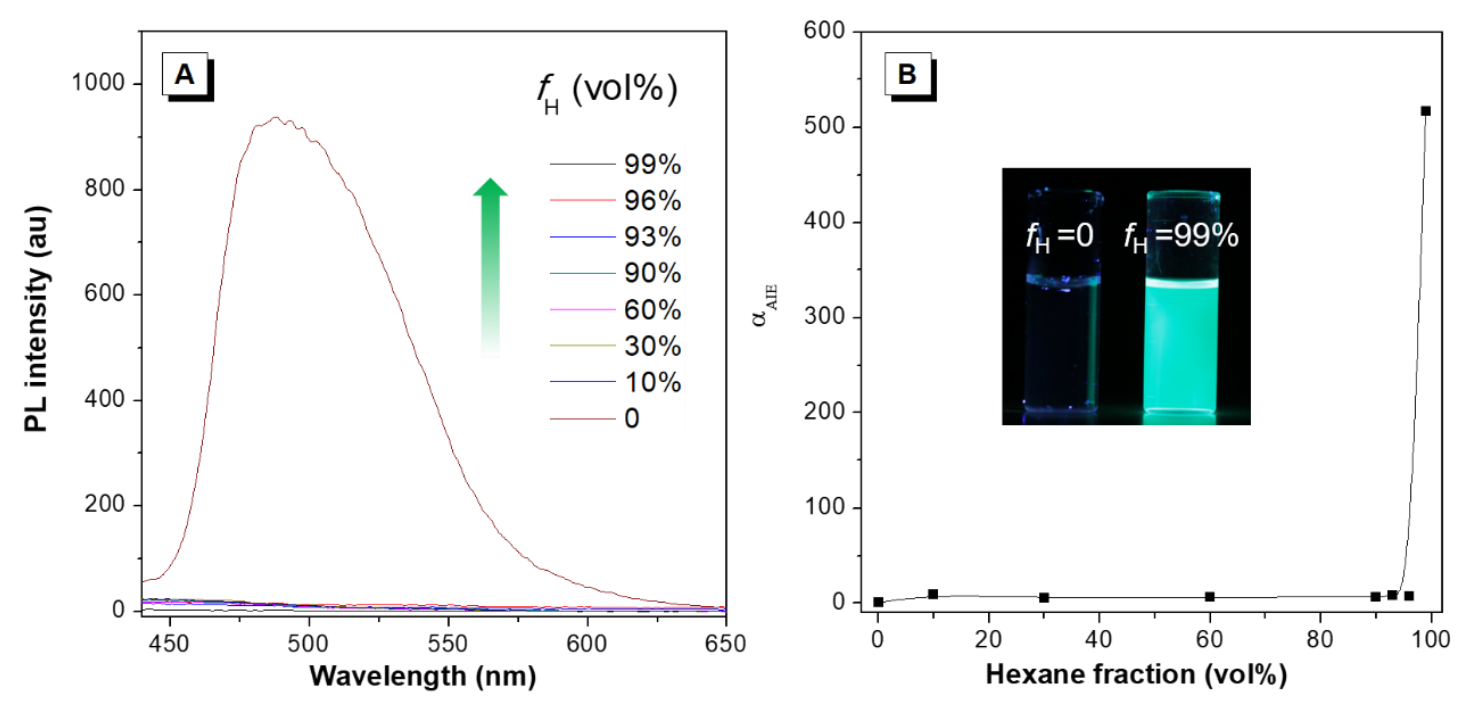

Figure 1. (A) PL spectra of $\mathrm{BaPt}(\mathrm{CN})_{4}$ in $\mathrm{H}_{2} \mathrm{O}$ /THF mixtures with different THF fractions $\left(f_{\mathrm{H}}\right)(2 \mu \mathrm{M})$. (B) The plot of the emission maximum and the relative emission intensity $\left(\alpha_{\mathrm{AIE}}=\mathrm{I} / \mathrm{I}_{0}\right)$ versus the composition of the aqueous mixture of $\mathrm{BaPt}(\mathrm{CN})_{4}$. Inset: Fluorescence photographs of $\mathrm{BaPt}(\mathrm{CN})_{4}$ in water and in $f_{\mathrm{H}}=99 \%$ mixture taken under 365 UV irradiation.

\section{Results and Discussion}

$\mathrm{BaPt}(\mathrm{CN})_{4}$ was purified by recrystallization three times before the optical properties measurement. Slow evaporation of the saturated aqueous solution of $\operatorname{BaPt}(\mathrm{CN})_{4}$ generates green crystals. These green crystals show brightly green emission with an emission maximum of $516 \mathrm{~nm}$ and a quantum yield of $47.9 \%$, while their dilute solution shows non-emission, suggesting their AIE activity. The AIE characteristic of $\mathrm{BaPt}(\mathrm{CN})_{4}$ is further demonstrated by studying its Photoluminescence (PL) behavior in the $\mathrm{H}_{2} \mathrm{O}$ /THF mixture (Fig. 1). In aqueous solution, $\mathrm{BaPt}(\mathrm{CN})_{4}$ is non-emissive, with adding THF into the aqueous solution until the THF fraction up to $99 \%$, molecules of $\mathrm{BaPt}(\mathrm{CN})_{4}$ begin to aggregate which turns on its emission. The aggregates at $99 \% \mathrm{THF}$ fraction was confirmed by DLS measurement, which gives a particle size of about 250 nm (Fig. S1). According to the AIE mechanism proposed by Tang and co-workers, the emission quenching in dilute was generally resulted by the active intramolecular motion. ${ }^{7}$ We thus speculated the emission quench of $\mathrm{BaPt}(\mathrm{CN})_{4}$ in aqueous solution was resulted by the active intramolecular vibration of the $\mathrm{CN}$ group of $\mathrm{Pt}(\mathrm{CN})_{4}{ }^{2-}$ since $\mathrm{CN}$ group shows strong vibrational signal in their IR spectra (Fig. S2).

To elucidate the role of intramolecular motion in contributing the AIE phenomenon of $\mathrm{BaPt}(\mathrm{CN})_{4}$, we measure its PL spectra in dilute solution $\left(2 \times 10^{-5} \mathrm{M}\right)$ at $77 \mathrm{~K}$, at which the intramolecular vibration will be greatly suppressed (Fig. S3). As expected, the dilute solution of $\mathrm{BaPt}(\mathrm{CN})_{4}$ show bright blue emission at $77 \mathrm{~K}$ with two emission peaks at $410 \mathrm{~nm}$ and $450 \mathrm{~nm}$, respectively. Interestingly, further increasing the concentration to $2 \times 10^{-4} \mathrm{M}$, a new emission peak at around $550 \mathrm{~nm}$ emerged, suggesting some aggregates seems formed. At a concentration of $2 \times 10^{-3} \mathrm{M}$, the shorter emission peaks disappeared and the emission peak at $550 \mathrm{~nm}$ show a slightly blue shift. These results indicate that when located at low temperature, the dye incline to aggregate possibly due to the 
decrease of the solubility. We also measured the UV spectra changes of $\operatorname{BaPt}(\mathrm{CN})_{4}$ solution with different concentrations at room temperature, which implies that even at room temperature, the dye has some aggregates since new absorption band emerge at a dye concentration of $2 \times 10^{-4} \mathrm{M} \cdot{ }^{38,39}$ However, at this concentration, the solution shows non-emission at room temperature. These results suggest that both the dye aggregates formed at low temperature and room temperature can influence their spectra wavelength, while only the dye aggregates formed at low temperature can emit brightly, which should be ascribed to the restriction of the intramolecular motion. And the nonemissive aggregates formed at room temperature possibly is due to the loose packing can't effectively restrict the molecular motion. It is worthy to note that Schindle and Viswanath et al. have studied the luminescence behavior of $\mathrm{BaPt}(\mathrm{CN})_{4}$ at $77 \mathrm{~K}$ much earlier and also observed the dye concentration depended emission wavelength. In their study, they assigned the longer wavelength emission to be dimer emission and cluster/oligomer emission, which is mainly resulted by the intermolecular Pt...Pt interaction. ${ }^{32,33}$ While they didn't discuss the influence of intramolecular motion on the emission property of the aggregates, which consequently can't explain the nonemissive characteristic of $\mathrm{BaPt}(\mathrm{CN})_{4}$ aggregates at room temperature reasonably.

To deeply understand this photophysical phenomenon, we analyzed the crystal structure of $\mathrm{BaPt}(\mathrm{CN})_{4}$. As shown in Fig. 2, the $\mathrm{Pt}(\mathrm{CN})_{4}{ }^{2-}$ show a square planar configuration without obvious rotation unit, suggesting intramolecular vibration should be the main molecular motion style. In addition, there is a strong Pt...Pt interaction exist in the crystal packing with a Pt...Pt distance of $3.319 \AA$. These Pt atoms can form orderly arranged Pt channel which should contribute greatly the wavelength red-shift of the aggregation. Additionally, multiple intermolecular hydrogen bonding $(\mathrm{H}-\mathrm{O}-\mathrm{H} \ldots \mathrm{N} \equiv \mathrm{C})$ and electrostatic interactions exist in the crystal which help rigidify the molecular structure, resulting the bright solid-state emission. Destroy these intermolecular interactions by grinding the crystal greatly decrease the emission intensity (Fig. S4). 
A



Side view

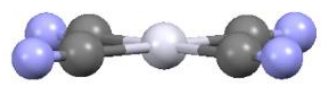

C

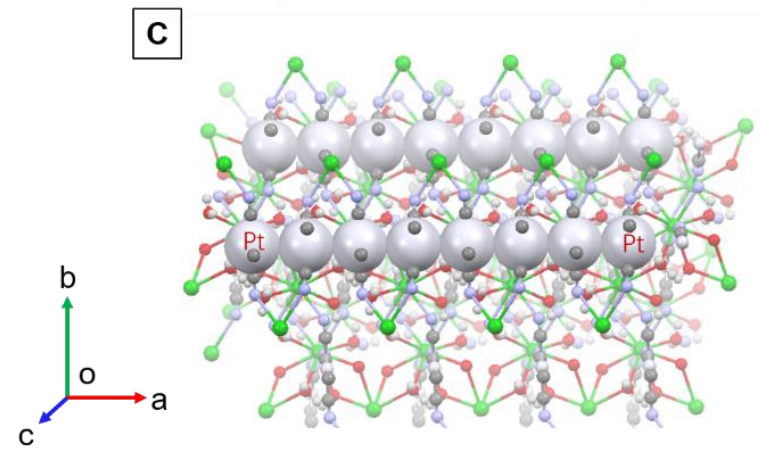

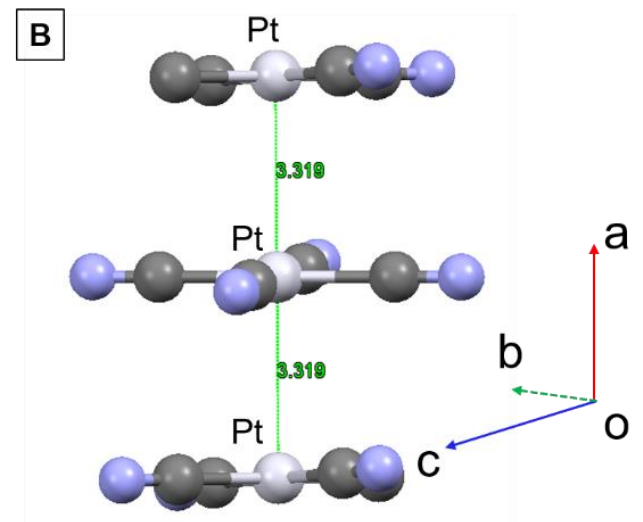



Figure 2. (A) Top-view and Side-view of the single crystal of $\operatorname{Pt}(\mathrm{CN})_{4}{ }^{2-}$. (B) Intermolecular Pt...Pt distance within the single crystal of $\mathrm{BaPt}(\mathrm{CN})_{4}$ with hiding the $\mathrm{Ba}^{2+}$ cation and $\mathrm{H}_{2} \mathrm{O}$ molecules. (C) The long-range orderly intermolecular Pt...Pt interactions within the single crystal of $\mathrm{BaPt}(\mathrm{CN})_{4}$. (D) Various intermolecular interactions in crystals of $\mathrm{BaPt}(\mathrm{CN})_{4}$.

Hydrostatic pressure depended PL measurement provide a suitable platform to study the influence of intermolecular interaction on the luminescence because it enables tuning the intermolecular distance through changing the hydrostatic pressure. . $^{8,40,41}$ Since the intermolecular Pt...Pt interactions possibly contribute greatly to the emission wavelength, we thus implemented the hydrostatic pressure experiment to investigate the PL changes upon high pressure. As shown in Fig. 3, within a pressure range from 0 $\mathrm{GPa}$ to $3.4 \mathrm{GPa}$, the luminescence intensity of $\mathrm{BaPt}(\mathrm{CN})_{4}$ crystal generally decrease while the emission wavelength gradually red-shifted from $516 \mathrm{~nm}$ to about $660 \mathrm{~nm}$, which is consistent well with the low temperature PL data. Therefore, the emission redshift of the high concentration aggregates relative to the low concentration aggregates is mainly due to the enhanced intermolecular Pt...Pt interactions. 

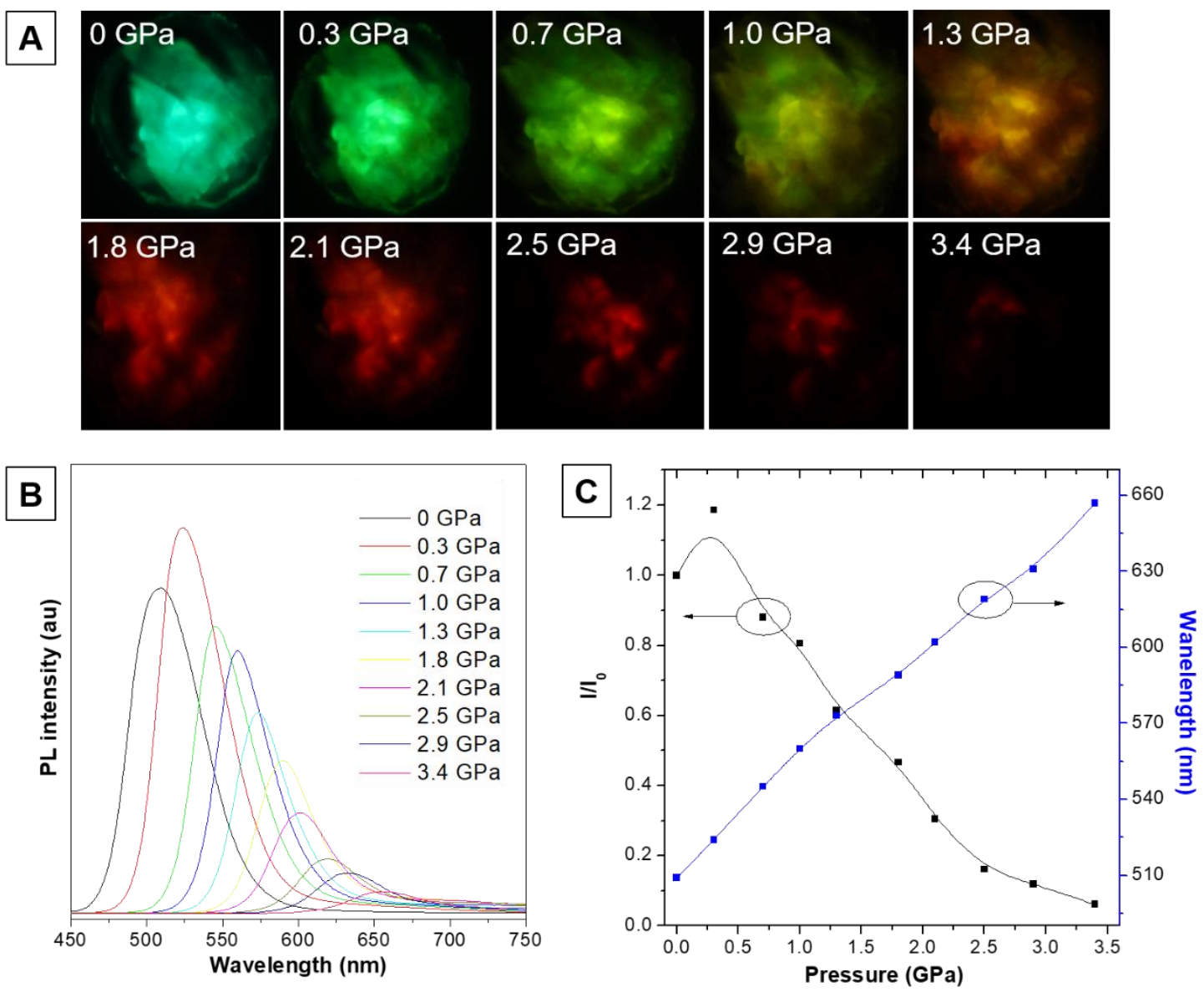

Figure 3. (A) Micrographs of the crystal of $\mathrm{BaPt}(\mathrm{CN})_{4}$ under varied hydrostatic pressure. (B) Emission spectra recorded during the compression process in the range of 0-3.4 GPa. (C) Plot of the PL intensity and emission wavelength vs. the hydrostatic pressure.
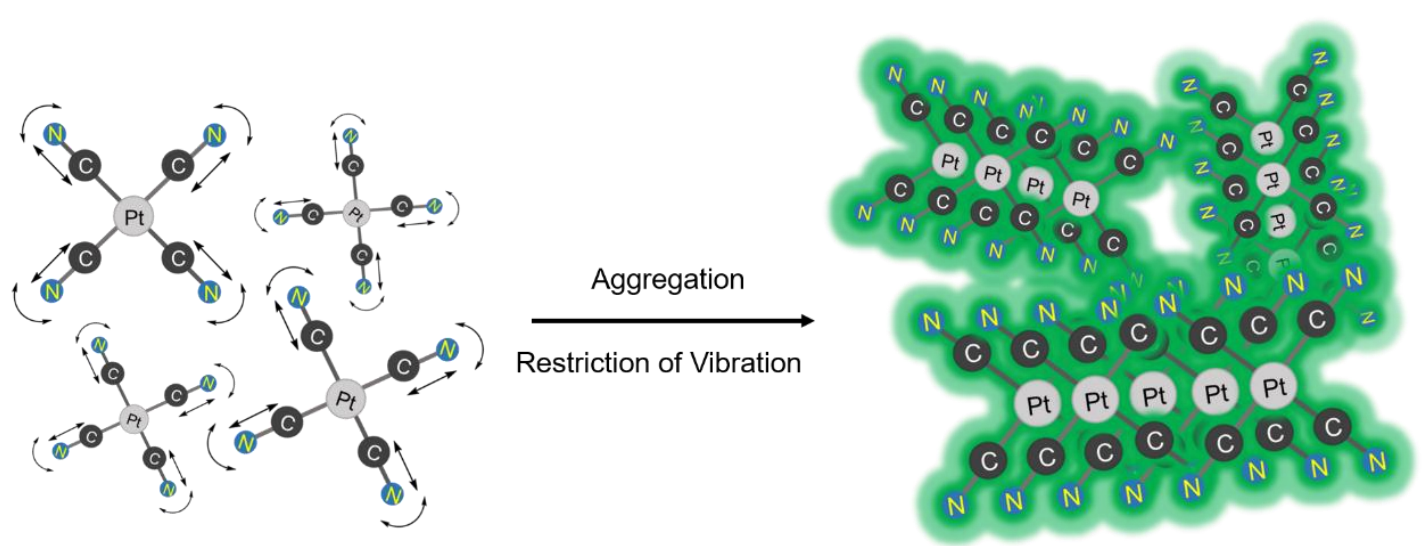

Based on these results, we propose a hypothetical diagram sketch which fit the data to describe the AIE process of $\mathrm{BaPt}(\mathrm{CN})_{4}$. In general, in solution state, $\mathrm{BaPt}(\mathrm{CN})_{4}$ exhibit active intramolecular vibration including stretching and swing upon photoirradiation, which accelerates the non-radiative decay of the exciton to quench the emission. While in the crystal, the multiple intermolecular interactions including hydrogen bonding, electrostatic interactions and Pt...Pt interactions help rigidify the molecular configuration and suppress the non-radiative decay, resulting the bright 
luminescence. Additionally, the intermolecular Pt...Pt interactions also contribute to decrease the energy gap between the HOMO and LUMO, benefiting the emission wavelength red shift of $\mathrm{BaPt}(\mathrm{CN})_{4}$ in aggregates, which also helps understanding the unconventional clusteroluminescence of some non-conjugated luminescent system.
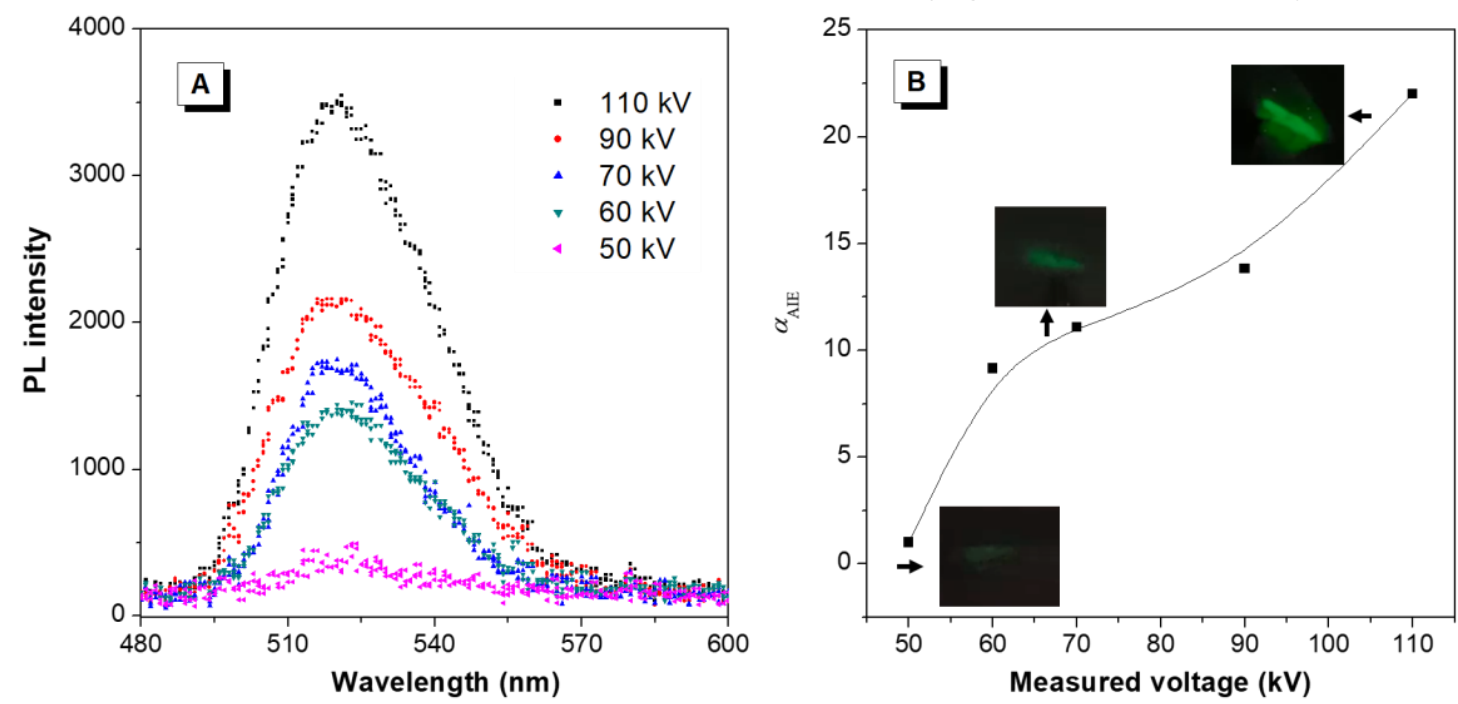

Figure 4. (A) PL spectra of $\mathrm{BaPt}(\mathrm{CN})_{4}$ under irradiation of $\mathrm{X}$-ray with varied dose by fiber optic spectrometer. (B) The plot of the relative emission intensity $\left(\alpha_{\text {AIE }}=I / I_{0}\right)$ versus the dose of X-ray. Inset: Fluorescence photographs of $\mathrm{BaPt}(\mathrm{CN})_{4}$ taken under varied X-ray irradiation.

Materials with X-ray luminescence are highly advantageous in improving the problem of auto-fluorescence and tissue penetration depth in vivo. ${ }^{17}$ However, most of the organic luminescent materials show poor absorption to X-ray, let alone the emission. According to the X-ray attenuation formula, molecules with high atomic number usually have strong absorption to X-ray, which can be used as CT imaging and X-ray luminescence imaging. We thus measured the response of $\operatorname{BaPt}(\mathrm{CN})_{4}$ to $\mathrm{X}$-ray irradiation since both $\mathrm{Ba}$ and $\mathrm{Pt}$ have large atomic number. The results indicate that in solution state, $\mathrm{BaPt}(\mathrm{CN})_{4}$ has no response to $\mathrm{X}$-ray irradiation, while in the solid state, $\mathrm{BaPt}(\mathrm{CN})_{4}$ show obvious X-ray luminescence whose intensity was enhanced with the increase the voltage of the X-ray. As a contrast, TPE shows no signal to X-ray irradiation. We further measured the response of $\mathrm{Na}_{2} \mathrm{Pt}(\mathrm{CN})_{4}$ and $\mathrm{K}_{2} \mathrm{Pt}(\mathrm{CN})_{4}$ to X-ray irradiation, it shows that the crystal of $\mathrm{K}_{2} \mathrm{Pt}(\mathrm{CN})_{4}$ show strong blue emission upon $\mathrm{X}$ ray excitation. Therefore, $\mathrm{Pt}$ atom possibly plays a more important role for the X-ray luminescence of $\mathrm{BaPt}(\mathrm{CN})_{4}$.

In summary, we have demonstrated the AIE property of $\mathrm{BaPt}(\mathrm{CN})_{4}$ for the first time, which can be extended to other $\operatorname{Pt}(\mathrm{CN})_{4}{ }^{2-}$ complexes. The underlying mechanism has been investigated which indicates that the intramolecular motion such as the vibration of the $\mathrm{CN}$ is responsible for the AIE phenomenon of $\mathrm{BaPt}(\mathrm{CN})_{4}$. Furthermore, the influence of the intermolecular Pt...Pt interaction on the emission wavelength has been well elucidated by concentration-depended PL measurement and hydrostatic depended PL measurement, which indicates that intermolecular Pt...Pt interaction contribute greatly to the emission wavelength red-shift of $\operatorname{BaPt}(\mathrm{CN})_{4}$. This to some extent helps 
understanding the unconventional clusteroluminescence of some non-conjugated luminescent system. More importantly, we found $\mathrm{BaPt}(\mathrm{CN})_{4}$ can be excited by X-ray, which fundamentally is highly significant since it can overcome the excitation limitation resulted auto-fluorescence and poor tissues penetration depth of AIEgens. To the best of our knowledge, this is the first reported AIEgen which could be excited by $\mathrm{X}$-ray. The current work possibly opens a new door for AIE research.

\section{Author Information}

Corresponding Authors

*E-mail: tangbenz@ust.hk

*E-mail: youhong.tang@flinders.edu.au

\# These authors contributed equally to this work.

\section{Acknowledgements}

This work was supported by the National Science Foundation of China (21788102 and 21674040), the Research Grants Council of Hong Kong (16308016, C6009-17G and A-HKUST605/16), the Innovation and Technology Commission (ITC-CNERC14SC01) and the Science and Technology Plan of Shenzhen (JCYJ20160229205601482 and JCYJ20180306174910791).

\section{Reference}

(1) Liu, Y.; Wolstenholme, C. H.; Carter, G. C.; Liu, H.; Hu, H.; Grainger, L. S.; Miao, K.; Fares, M.; Hoelzel, C. A.; Yennawar, H. P.; Ning, G.; Du, M.; Bai, L.; Li, X.; Zhang, X., Modulation of Fluorescent Protein Chromophores To Detect Protein Aggregation with Turn-On Fluorescence. J. Am. Chem. Soc. 2018, 140, 7381-7384.

(2) Fu, W.; Yan, C.; Guo, Z.; Zhang, J.; Zhang, H.; Tian, H.; Zhu, W.-H., Rational Design of NearInfrared Aggregation-Induced-Emission-Active Probes: In Situ Mapping of Amyloid- $\beta$ Plaques with Ultrasensitivity and High-Fidelity. J. Am. Chem. Soc. 2019, 141, 3171-3177.

(3) Qian, H.; Cousins, M. E.; Horak, E. H.; Wakefield, A.; Liptak, M. D.; Aprahamian, I., Suppression of Kasha's rule as a mechanism for fluorescent molecular rotors and aggregationinduced emission. Nat. Chem. 2016, 9, 83

(4) Liu, S.; Zhou, X.; Zhang, H.; Ou, H.; Lam, J. W. Y.; Liu, Y.; Shi, L.; Ding, D.; Tang, B. Z., Molecular Motion in Aggregates: Manipulating TICT for Boosting Photothermal Theranostics. J. Am. Chem. Soc. 2019, 141, 5359-5368.

(5) Zhao, Z.; Chen, C.; Wu, W.; Wang, F.; Du, L.; Zhang, X.; Xiong, Y.; He, X.; Cai, Y.; Kwok, R. T. K.; Lam, J. W. Y.; Gao, X.; Sun, P.; Phillips, D. L.; Ding, D.; Tang, B. Z., Highly efficient photothermal nanoagent achieved by harvesting energy via excited-state intramolecular motion within nanoparticles. Nat. Commun. 2019, 10, 768.

(6) Zhu, C.; Kwok, R. T. K.; Lam, J. W. Y.; Tang, B. Z., Aggregation-Induced Emission: A Trailblazing Journey to the Field of Biomedicine. ACS Appl. Bio Mater. 2018, 1, 1768-1786.

(7) Chen, Y.; Lam, J. W. Y.; Kwok, R. T. K.; Liu, B.; Tang, B. Z., Aggregation-induced emission: fundamental understanding and future developments. Mater. Horiz. 2019, 6, 428-433.

(8) Zhao, Z.; Zheng, X.; Du, L.; Xiong, Y.; He, W.; Gao, X.; Li, C.; Liu, Y.; Xu, B.; Zhang, J.; Song, F.; Yu, Y.; Zhao, X.; Cai, Y.; He, X.; Kwok, R. T. K.; Lam, J. W. Y.; Huang, X.; Lee Phillips, D.; Wang, H.; Tang, B. Z., Non-aromatic annulene-based aggregation-induced 
emission system via aromaticity reversal process. Nat. Commun. 2019, 10, 2952.

(9) La, D. D.; Bhosale, S. V.; Jones, L. A.; Bhosale, S. V., Tetraphenylethylene-Based AIE-Active Probes for Sensing Applications. ACS Appl. Mater. \& Interfaces 2018, 10, 12189-12216.

(10) Hu, F.; Mao, D.; Kenry; Cai, X.; Wu, W.; Kong, D.; Liu, B., A Light-Up Probe with Aggregation-Induced Emission for Real-Time Bio-orthogonal Tumor Labeling and ImageGuided Photodynamic Therapy. Angew. Chem. Inter. Ed. 2018, 57, 10182-10186.

(11) Zhan, C.; You, X.; Zhang, G.; Zhang, D., Bio-/Chemosensors and Imaging with AggregationInduced Emission Luminogens. Chem. Rec. 2016, 16, 2142-2160.

(12) Feng, G.; Yuan, Y.; Fang, H.; Zhang, R.; Xing, B.; Zhang, G.; Zhang, D.; Liu, B., A light-up probe with aggregation-induced emission characteristics (AIE) for selective imaging, nakedeye detection and photodynamic killing of Gram-positive bacteria. Chem. Commun. 2015, 51, 12490-12493.

(13) Wang, D.; Lee, M. M. S.; Shan, G.; Kwok, R. T. K.; Lam, J. W. Y.; Su, H.; Cai, Y.; Tang, B. Z., Highly Efficient Photosensitizers with Far-Red/Near-Infrared Aggregation-Induced Emission for In Vitro and In Vivo Cancer Theranostics. Adv. Mater. 2018, 30, 1802105.

(14) Shao, A.; Xie, Y.; Zhu, S.; Guo, Z.; Zhu, S.; Guo, J.; Shi, P.; James, T. D.; Tian, H.; Zhu, W.H., Far-Red and Near-IR AIE-Active Fluorescent Organic Nanoprobes with Enhanced TumorTargeting Efficacy: Shape-Specific Effects. Angew. Chem. Inter. Ed. 2015, 54, 7275-7280.

(15) Liu, L.-J.; Liu, W.; Ji, G.; Wu, Z.-Y.; Xu, B.; Qian, J.; Tian, W.-J., NIR Emission Nanoparticles Based on FRET Composed of AIE Luminogens and NIR Dyes for Two-photon Fluorescence Imaging. Chinese J.Polym. Sci. 2019, 37, 401-408.

(16) Qi, J.; Chen, C.; Ding, D.; Tang, B. Z., Aggregation-Induced Emission Luminogens: Union Is Strength, Gathering Illuminates Healthcare. Adv. Healthcare Mater. 2018, 7, 1800477.

(17) Chen, X.; Song, J.; Chen, X.; Yang, H., X-ray-activated nanosystems for theranostic applications. Chem. Soc. Rev. 2019, 48, 3073-3101.

(18) Osakada, Y.; Pratx, G.; Hanson, L.; Solomon, P. E.; Xing, L.; Cui, B., X-ray excitable luminescent polymer dots doped with an iridium(iii) complex. Chem. Commun. 2013, 49, 43194321.

(19) Naczynski, D. J.; Sun, C.; Türkcan, S.; Jenkins, C.; Koh, A. L.; Ikeda, D.; Pratx, G.; Xing, L., X-ray-Induced Shortwave Infrared Biomedical Imaging Using Rare-Earth Nanoprobes. Nano Lett. 2015, 15, 96-102.

(20) Chen, Q.; Wu, J.; Ou, X.; Huang, B.; Almutlaq, J.; Zhumekenov, A. A.; Guan, X.; Han, S.; Liang, L.; Yi, Z.; Li, J.; Xie, X.; Wang, Y.; Li, Y.; Fan, D.; Teh, D. B. L.; All, A. H.; Mohammed, O. F.; Bakr, O. M.; Wu, T.; Bettinelli, M.; Yang, H.; Huang, W.; Liu, X., All-inorganic perovskite nanocrystal scintillators. Nature 2018, 561, 88-93.

(21) Kirakci, K.; Fejfarová, K.; Martinčík, J.; Nikl, M.; Lang, K., Tetranuclear Copper(I) Iodide Complexes: A New Class of X-ray Phosphors. Inorg. Chem. 2017, 56, 4609-4614.

(22) Kirakci, K.; Zelenka, J.; Rumlová, M.; Martinčík, J.; Nikl, M.; Ruml, T.; Lang, K., Octahedral molybdenum clusters as radiosensitizers for X-ray induced photodynamic therapy. J. Mater. Chem. B 2018, 6, 4301-4307.

(23) Nikl, M.; Yoshikawa, A., Recent R\&D Trends in Inorganic Single-Crystal Scintillator Materials for Radiation Detection. Adv. Optical Mater. 2015, 3, 463-481.

(24) Osakada, Y.; Pratx, G.; Sun, C.; Sakamoto, M.; Ahmad, M.; Volotskova, O.; Ong, Q.; Teranishi, T.; Harada, Y.; Xing, L.; Cui, B., Hard X-ray-induced optical luminescence via 
biomolecule-directed metal clusters. Chem. Commun. 2014, 50, 3549-3551.

(25) Li, X.; Xue, Z.; Jiang, M.; Li, Y.; Zeng, S.; Liu, H., Soft X-ray activated NaYF4:Gd/Tb scintillating nanorods for in vivo dual-modal X-ray/X-ray-induced optical bioimaging. Nanoscale 2018, 10, 342-350.

(26) Wong, K. M.-C.; Hui, C.-K.; Yu, K.-L.; Yam, V. W.-W., Luminescence studies of dinuclear platinum(II) alkynyl complexes and their mixed-metal platinum(II)-copper(I) and -silver(I) complexes. Coord. Chem. Rev. 2002, 229, 123-132.

(27) Zuleta, J. A.; Burberry, M. S.; Eisenberg, R., Platinum(II) diimine dithiolates. New solution luminescent complexes. Coord. Chem. Rev. 1990, 97, 47-64.

(28) Chan, C.-W.; Cheng, L.-K.; Che, C.-M., Luminescent donor-acceptor platinum(ii) complexes. Coord. Chem. Rev. 1994, 132, 87-97.

(29) Williams, J. A. G.; Beeby, A.; Davies, E. S.; Weinstein, J. A.; Wilson. C. An Alternative Route to Highly Luminescent Platinum(II) Complexes: Cyclometalation with $\mathrm{N} \wedge \mathrm{C} \wedge \mathrm{N}$-Coordinating Dipyridylbenzene Ligands. Inorg. Chem. 2003, 42, 8609-8611.

(30) Mauro, M.; Aliprandi, A.; Septiadi, D.; Kehr, N. S.; De Cola, L., When self-assembly meets biology: luminescent platinum complexes for imaging applications. Chem. Soc. Rev. 2014, 43, 4144-4166.

(31) Stokes George, G., XVI. On the change of refrangibility of light.-No. II. Philos. Trans. R. Soc. London, 1853, 143, 385-396.

(32) Schindler, J. W.; Fukuda, R. C.; Adamson, A. W. Photophysics of Aqueous Pt(CN) $4^{2-} . J . A m$. Chem. Soc. 1982, 104, 3596-3600.

(33) Viswanath, A. K.; Krogh-Jespersen, M. B.; Vetuskey, J.; Baker, C.; Ellenson, W. D.; Patterson, H. H., Laser excited luminescence and optical absorption studies of interacting tetracyanoplatinate ions in crystals, pellets, and frozen aqueous solutions. Mol. Phys. 1981, 42, 1431-1451.

(34) Sun, Y.; Ye, K.; Zhang, H.; Zhang, J.; Zhao, L.; Li, B.; Yang, G.; Yang, B.; Wang, Y.; Lai, S.-


Interactions. Angew. Chem. Inter. Ed. 2006, 45, 5610-5613.

(35) Zhao, S.; Yu, F.; Yang, G.; Zhang, H.; Su, Z.; Wang, Y., Theoretical study on the charge transport property of $\mathrm{Pt}(\mathrm{CNtBu}) 2(\mathrm{CN}) 2$ nanowires induced by $\mathrm{Pt} \cdots \mathrm{Pt}$ interactions. Dalton Trans., 2012, 41, 7272-7277.

(36) Wenger, O. S., Vapochromism in Organometallic and Coordination Complexes: Chemical Sensors for Volatile Organic Compounds. Chem. Rev. 2013, 113, 3686-3733.

(37) Varju, B. R.; Wollschlaeger, S. A.; Leznoff, D. B., Zinc Tetracyanoplatinate: A Reversible Luminescence-Based Ammonia Sensor. Chem. Eur. J. 2019, 25, 9017-9025.

(38) Rawashdeh-Omary, M. A.; Omary, M. A.; Patterson, H. H., Oligomerization of $\mathrm{Au}(\mathrm{CN})^{2-}$ and $\mathrm{Ag}(\mathrm{CN})^{2-}$ Ions in Solution via Ground-State Aurophilic and Argentophilic Bonding. J. Am. Chem. Soc. 2000, 122, 10371-10380.

(39) Rawashdeh-Omary, M. A.; Omary, M. A.; Patterson, H. H.; Fackler, J. P., Excited-State Interactions for $\left[\mathrm{Au}(\mathrm{CN})^{2-}\right] \mathrm{n}$ and $\left[\mathrm{Ag}(\mathrm{CN})^{2-}\right] \mathrm{n}$ Oligomers in Solution. Formation of Luminescent Gold-Gold Bonded Excimers and Exciplexes. J. Am. Chem. Soc. 2001, 123, 11237-11247.

(40) Qi, Q.; Qian, J.; Tan, X.; Zhang, J.; Wang, L.; Xu, B.; Zou, B.; Tian, W., Remarkable TurnOn and Color-Tuned Piezochromic Luminescence: Mechanically Switching Intramolecular 
Charge Transfer in Molecular Crystals. Adv. Funct. Mater. 2015, 25, 4005-4010.

(41) Yang, Z.; Chi, Z.; Mao, Z.; Zhang, Y.; Liu, S.; Zhao, J.; Aldred, M. P.; Chi, Z., Recent advances in mechano-responsive luminescence of tetraphenylethylene derivatives with aggregation-induced emission properties. Mater. Chem. Front. 2018, 2, 861-890. 